\title{
Modeling the Extremely Lightweight Zerodur Mirror (ELZM) thermal soak test
}

\author{
Thomas E. Brooks*a, Ron Eng ${ }^{\mathrm{a}}$, Tony Hull ${ }^{\mathrm{b}}$, H. Philip Stahl ${ }^{\mathrm{a}}$ \\ ${ }^{a}$ NASA's Marshall Space Flight Center, AL 35812; \\ ${ }^{\mathrm{b}}$ University of New Mexico, Albuquerque, NM 87131
}

\begin{abstract}
Exoplanet science requires extreme wavefront stability (10 pm change/10 minutes), so every source of wavefront error (WFE) must be characterized in detail. This work illustrates the testing and characterization process that will be used to determine how much surface figure error (SFE) is produced by mirror substrate materials' CTE distributions. Schott's extremely lightweight Zerodur mirror (ELZM) was polished to a sphere, mounted, and tested at Marshall Space Flight Center (MSFC) in the X-Ray and Cryogenic Test Facility (XRCF). The test transitioned the mirror's temperature from an isothermal state at $292 \mathrm{~K}$ to isothermal states at $275 \mathrm{~K}, 250 \mathrm{~K}$ and $230 \mathrm{~K}$ to isolate the effects of the mirror's CTE distribution. The SFE was measured interferometrically at each temperature state and finite element analysis (FEA) has been completed to assess the predictability of the change in the mirror's surface due to a change in the mirror's temperature. The coefficient of thermal expansion (CTE) distribution in the ELZM is unknown, so the analysis has been correlated to the test data. The correlation process requires finding the sensitivity of SFE to a given CTE distribution in the mirror. A novel hand calculation is proposed to use these sensitivities to estimate thermally induced SFE. The correlation process was successful and is documented in this paper. The CTE map that produces the measured SFE is in line with the measured data of typical boules of Schott's Zerodur glass.
\end{abstract}

Keywords: CTE homogeneity, mirror, thermal soak, thermal expansion, Zerodur, surface figure error, finite element analysis, CTE

\section{INTRODUCTION}

Exoplanet science requires extreme wavefront stability (10 pm change/10 minutes), so every source of wavefront error (WFE) must be characterized in detail. The SFE caused by other sources must be eliminated in order to observe only the effects of the CTE distribution. When mounted on a kinematic mount, a mirror will bend as its temperature changes because of 1) thermal gradients and 2) the mirror's CTE gradients. The ELZM was tested at four nearly isothermal states to isolate the effects of CTE gradients by minimizing the bending from the thermal gradients. The mirror's mount is exceedingly compliant to improve the kinematics. The ELZM mirror is a $1.2 \mathrm{~m}$ aperture, $3.1 \mathrm{~m}$ radius of curvature, isogrid, Zerodur mirror that is $88 \%$ lightweighted. This paper presents the finite element analysis of the test setup and provides SFE sensitivities to several CTE distributions. The SFE caused by an isothermal soak and a CTE distribution is the same as the SFE caused by a mirror having the CTE of the isothermal soak's change in temperature and a thermal gradient having the same shape and amplitude as the CTE distribution (explained further in section 5.1). Because this is true, this work may help the reader obtain an intuition for a temperature distribution's impact on the mirror's surface figure error (examples in section 5.2).

\section{TEST SETUP AND DATA OVERVIEW}

The mirror is tested using a center of curvature test while in the XRCF's large vacuum chamber. The mirror is $1.2 \mathrm{~m}$ in diameter and the $1-\mathrm{m}$ clear aperture is measured. The interferometer is in a pressure tight enclosure with an optical window between the interferometer and the ELZM as shown in Figure 1. An image of the mirror on the test stand in the XRCF is shown in Figure 2. Thermal diodes were placed on the mirror and its mount to monitor temperatures. The mirror's surface is measured at room temperature $(292 \mathrm{~K}), 275 \mathrm{~K}, 250 \mathrm{~K}$ and $230 \mathrm{~K}$ with the mirror in a nearly isothermal state as evidenced by the thermal measurements on the graph in Figure 3. In total, there are 40 thermal diodes on the mirror and its mount.

*thomas.brooks@nasa.gov;

(256)797-3147;

nasa.gov 


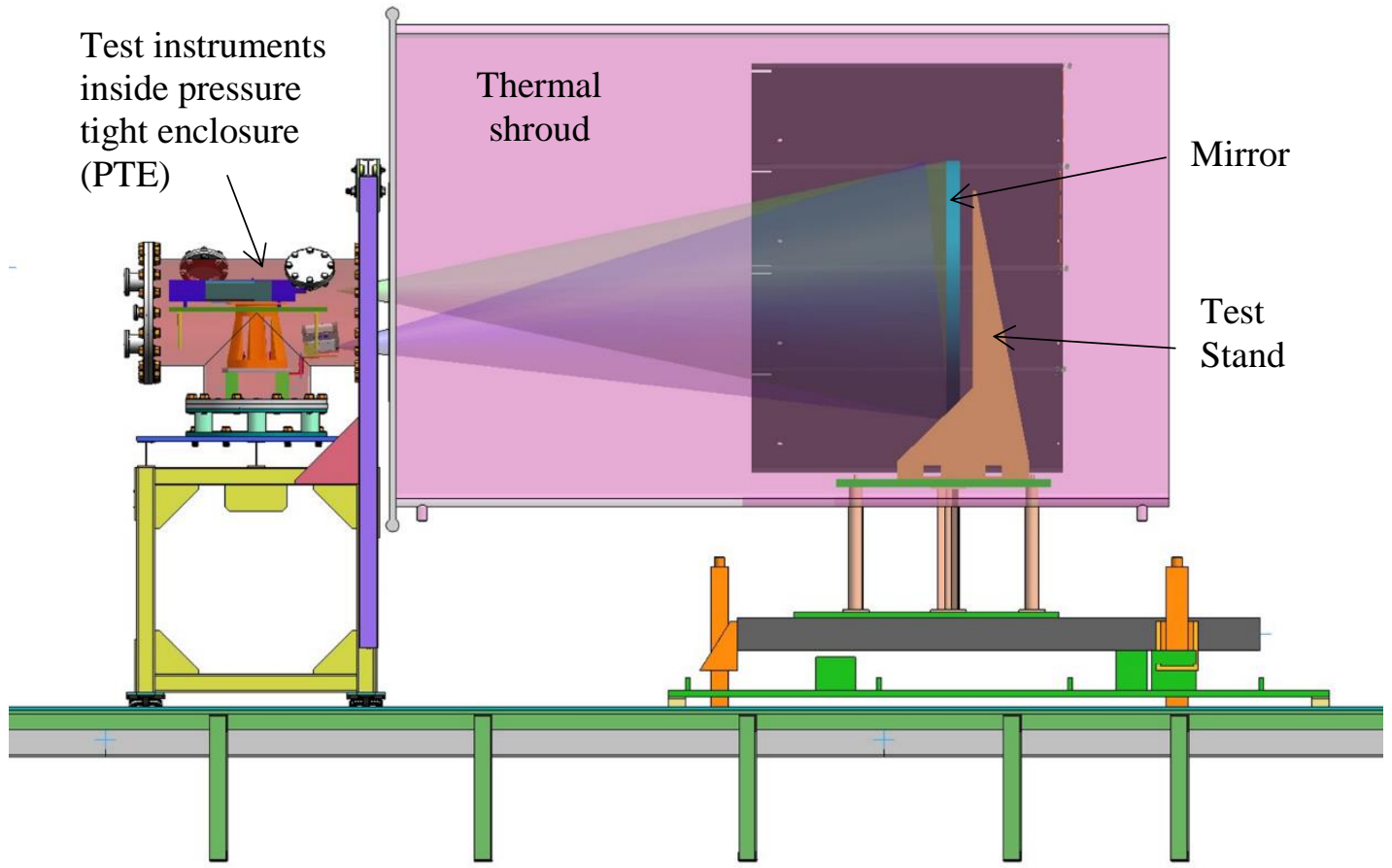

Figure 1: Test Setup: The mirror sits inside of the XRCF's large vacuum chamber, and it is supported by a kinematic hexapod mount with a low CTE back structure. The back structure kinematically mounts to the test stand using cone-vee-flat blocks and tooling balls. An interferometer is placed at the mirror's center of curvature and the surface figure error of the mirror is measured with a repeatability of $\sim 6 \mathrm{~nm}$. An infrared camera is used to measure the thermal emission of the mirror's front face, and this data can be processed to gain an indirect measurement the mirror's temperature distribution. Thermal diodes are located on the mirror and its mount to ensure that the mirror is isothermal at every SFE measurement.

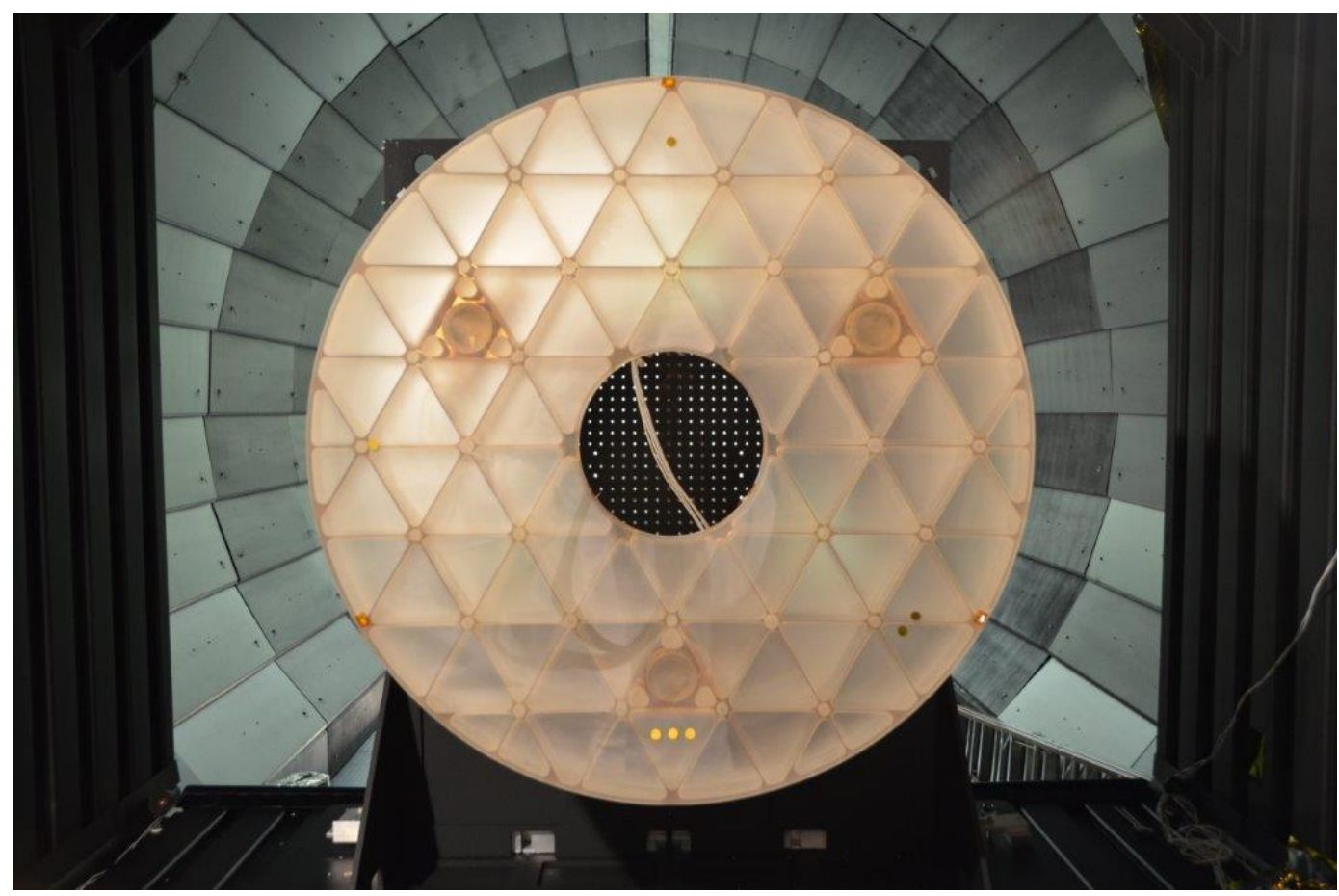

Figure 2: The ELZM is shown (from the interferometer's perspective) mounted in the XRCF's large cryogenic chamber. 


\subsection{Test Data}

Temperature measurements were taken continuously at 21 locations on the mirror throughout the test. Some of the diode locations may be seen in Figure 3. The mirror's average temperature, maximum temperature, minimum temperature, and temperature gradient are graphed versus time in Figure 4. Vertical blue lines indicate the times at which interferograms were taken. The SFE measured at $230 \mathrm{~K}, 250 \mathrm{~K}$, and $275 \mathrm{~K}$ was subtracted by the SFE measured at room temperature to result in the "delta maps" shown in Figure 5. Thermal expansion of the mirror's mount and realignment of the interferometer resulted in interferometric shearing which is most evident in the $230 \mathrm{~K}$ and $250 \mathrm{~K}$ delta maps. The interferometric shearing has been filtered out using a process described in section 2.2.
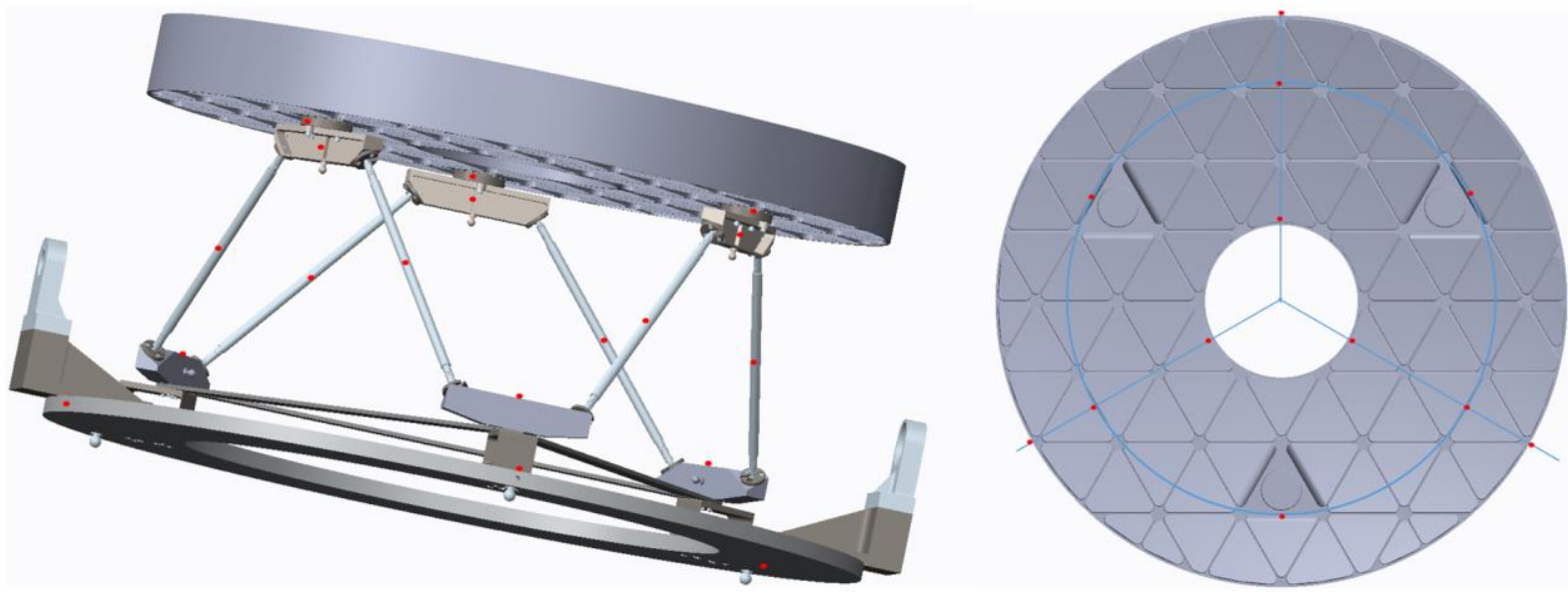

Figure 3: Location of thermal diodes on the mirror and the mount. Not all diodes are visible in this image. In total, 40 diodes measure temperatures on the mirror and mount.

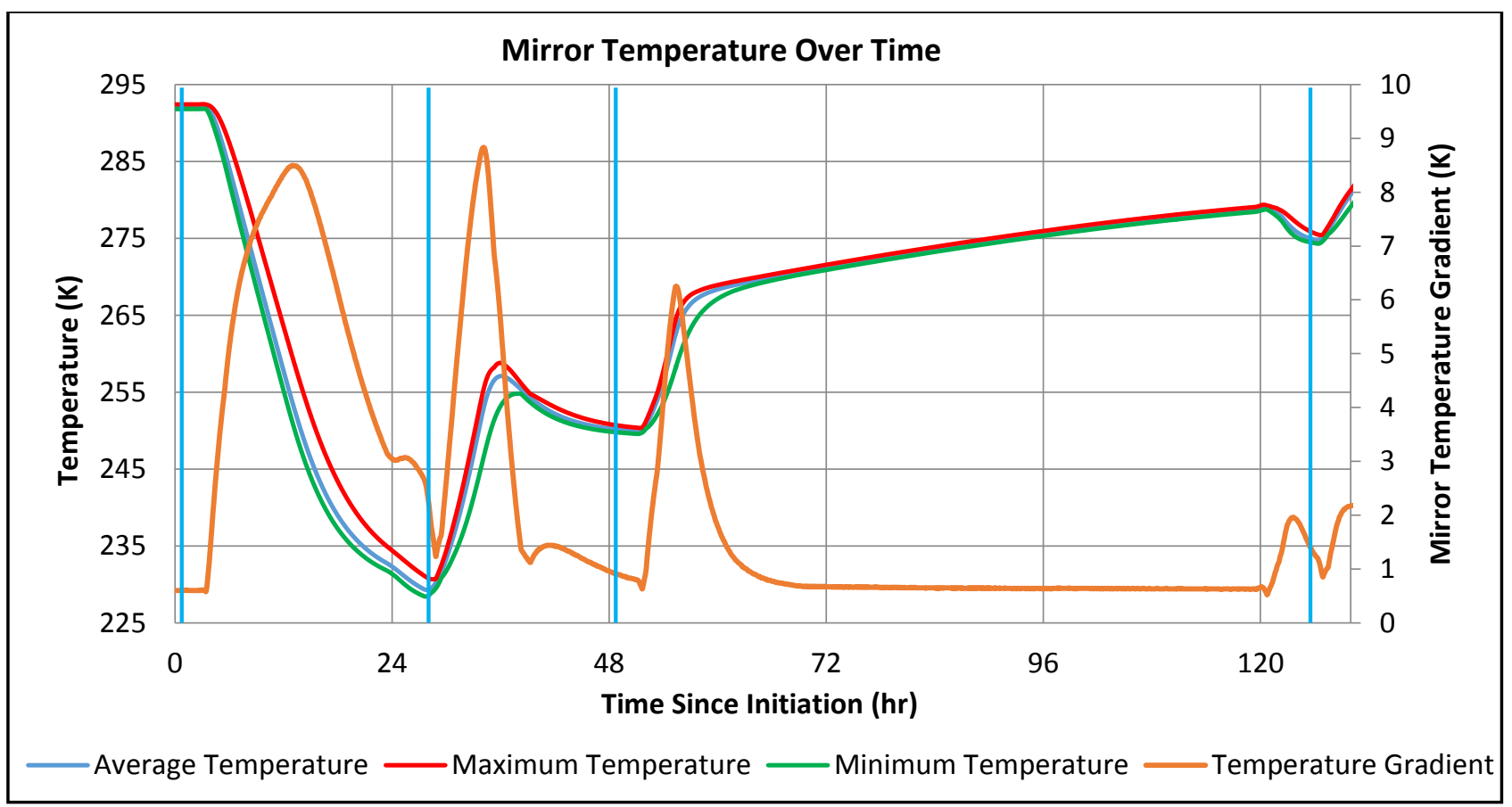

Figure 4: Temperature Data over One Cycle. The left axis indicates the mirror's maximum, minimum and average temperatures. The right axis indicates the maximum minus the minimum temperature (commonly called a temperature gradient). A small bias between thermocouples puts an artificial floor on the temperature gradient. The vertical, light blue lines indicate the times at which the mirror's surface figure is measured. 
The peak to valley $(\mathrm{P}-\mathrm{V})$ of the mirror's temperature distribution is very small at every SFE measurement with the least isothermal measurement occurring at the $230 \mathrm{~K}$ where there is a $2.5 \mathrm{~K}$ temperature gradient in the mirror. Analysis indicates that such a small temperature gradient will have a negligible impact on the mirror's SFE because of the thermal gradient's shape and the low average CTE of the mirror. Therefore, most of the measured SFE is being caused by CTE gradients.

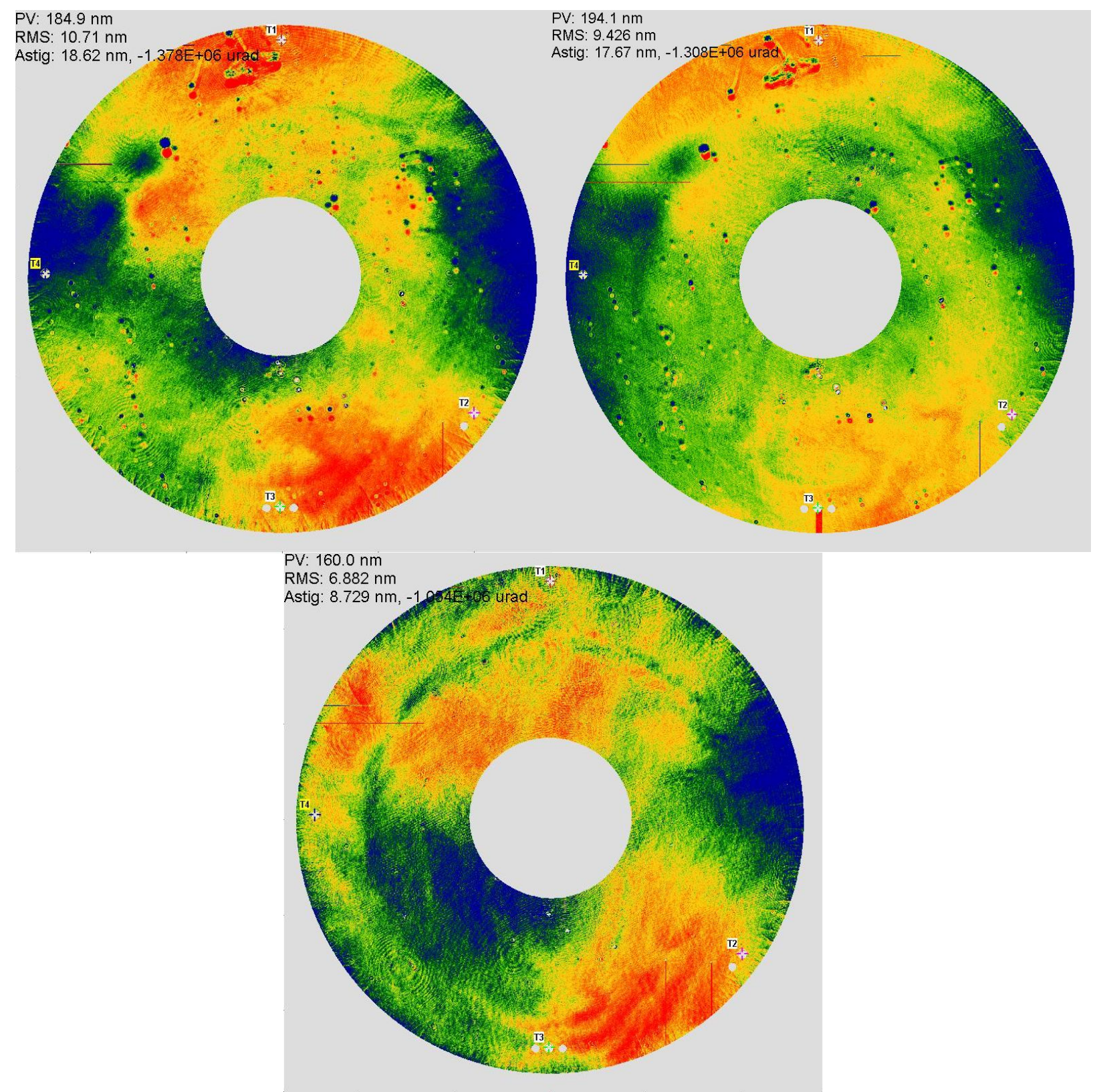

Figure 5: Top left: Surface at $230 \mathrm{~K}$ minus surface at $292 \mathrm{~K}$. Top Right: Surface at $250 \mathrm{~K}$ minus surface at 292K. Bottom: Surface at $275 \mathrm{~K}$ minus surface at $292 \mathrm{~K}$

\subsection{Test Data Filtering Process}

Interferometric shearing is clearly apparent in two of the delta maps shown in Figure 5. Some small fraction of the interferometer's many pixels are biased and measure SFEs that are significantly different than the rest of the pixels. This interferometric shearing is caused by the mirror moving relative to the interferometer, so when the delta map is taken, the 
erroneous pixels appear to move on the mirror's surface and do not cancel themselves out during subtraction. It can be seen in Figure 5 as very high surface measurement circles (red) below and to the right of very low surface measurement circles (blue). This error source is difficult and unnecessary to replicate in the analysis, so it is simply removed by filtering. Unfortunately, many of the erroneous measurements caused by this interferometric shearing do not exceed the bounds of truly measured surface figure errors, so they cannot be filtered out by putting maximum and minimum bounds on the measurement. A gradient method is used to filter the measurement instead of a range filtering technique. The gradient filtering technique is described graphically in Figure 6. A comparison between the original, unfiltered 230K delta map and the filtered 230K delta map is shown in Figure 7.

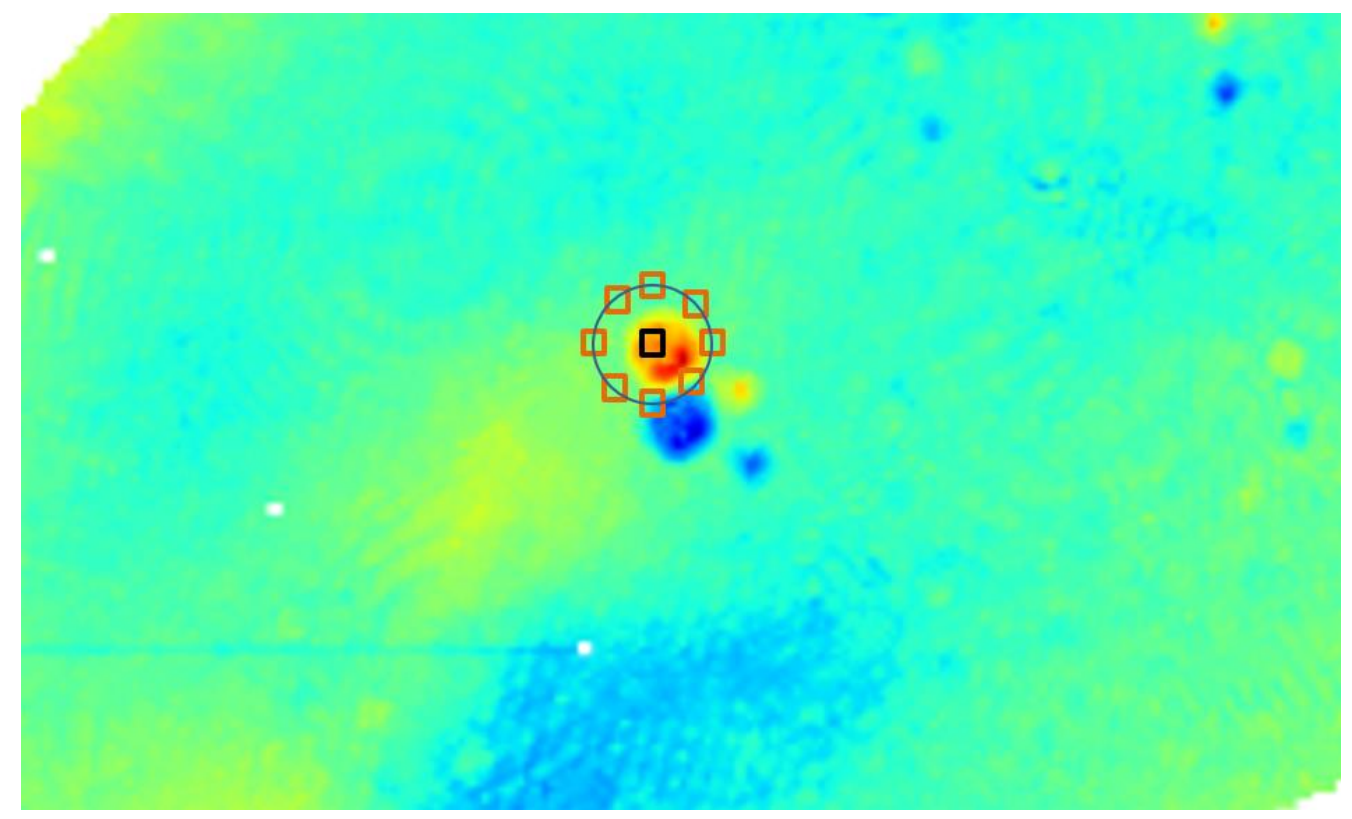

Figure 6: Filtering Example. The black square represents a pixel that is being tested for filtering, and the orange squares represent pixels which the black pixel is compared against. If the difference between the black pixel's value and the orange pixels' values is too great for half or more of the pixel differences, then the black pixel is filtered out. The distance between the black square and every orange square is the same, so the difference in the surface measured at black and orange pixels is effectively a SFE gradient.
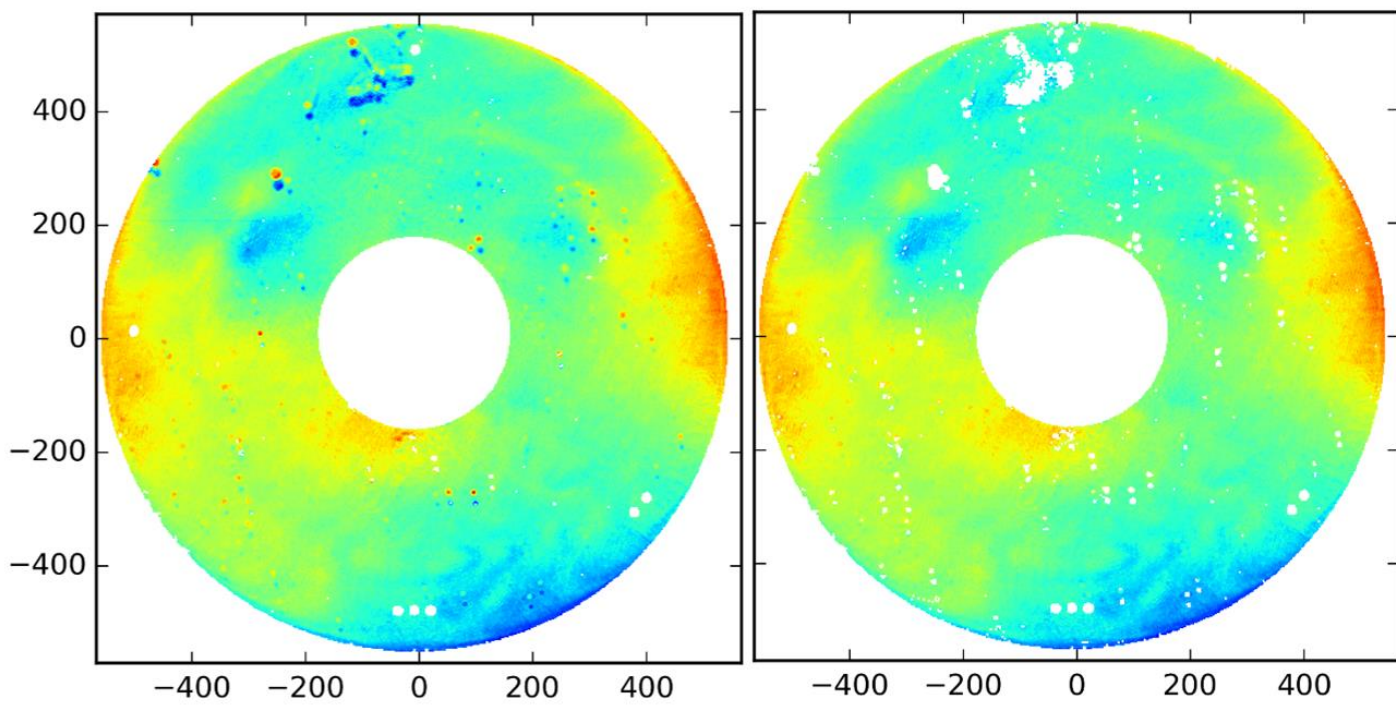

$8.15 \mathrm{e}-02$

$6.13 \mathrm{e}-02$

$4.12 \mathrm{e}-02$

2.10e-02

8.40e-04

$-1.93 \mathrm{e}-02$

$-3.95 \mathrm{e}-02$ 嵌

$-5.97 e-02$

$-7.98 \mathrm{e}-02$

$-1.00 \mathrm{e}-01$

Figure 7: Comparison between filtered and unfiltered data. The left figure shows a raw 292-230K delta map without filtering. The right image shows what the data looks like after filtering is performed. 


\section{MODELING APPROACH}

A finite element model (FEM) was made of the mirror and mount using NASTRAN. Images of this FEM are in

Figure 8. The displacements from the NASTRAN analysis were input into a SigFit analysis to determine the mirror's SFE (with defocus removed). Defocus was removed, because the interferometer is put into focus to take the measurement. Initial analyses included both the mirror and the mount and concluded that the mount's effect on the mirror's SFE was relatively small, so the mount was subsequently removed to reduce run time. Analysis was done to determine the magnitude of the effect of thermal gradients. Temperature measurements as well as finite element analysis culminated in the conclusion that thermal gradients will not have a sizeable effect on the mirror's SFE. CTE distributions were placed on the mirror in order to simulate the SFE caused by CTE gradients. The CTE distribution of the mirror was not known in advance, so several CTE distributions were placed on the mirror to bound the effect. Schott provides an example CTE distribution in their catalogue and that distribution is shown in Figure 9. ${ }^{[1]}$

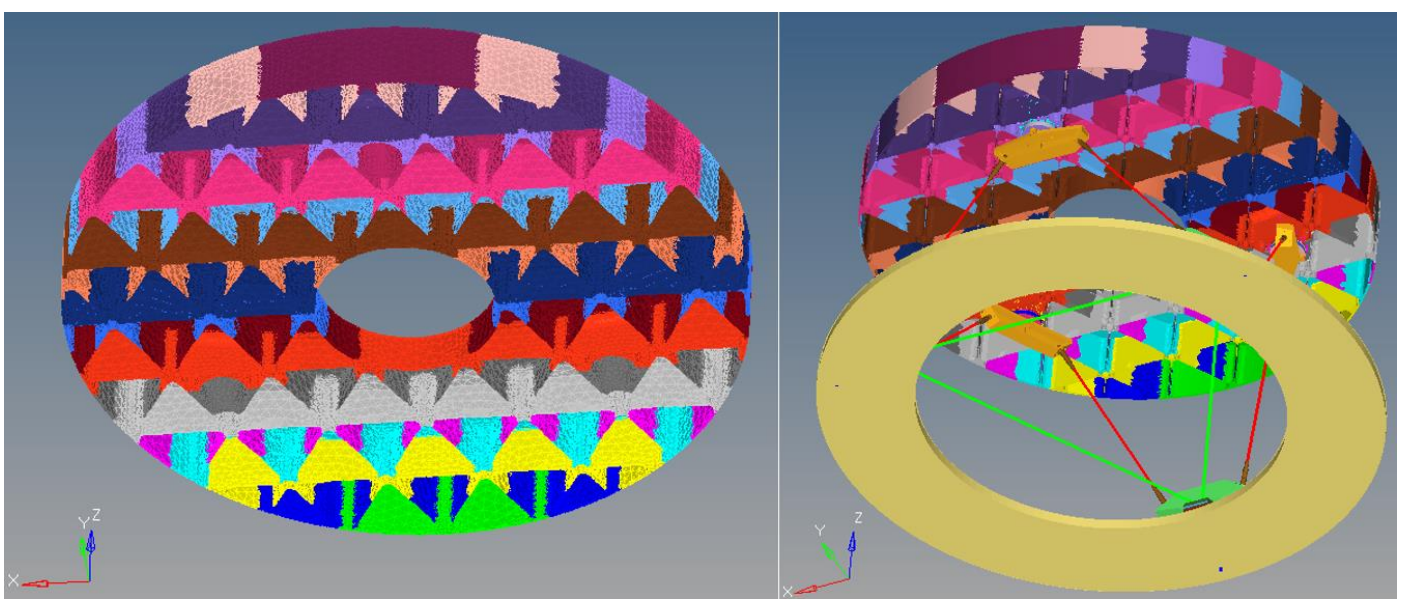

Figure 8: Left: An image of the FEM is shown with all things hidden except the mirror. The mirror has a lateral (tip/tilt) CTE distribution. Right: An image of the FEM is shown that includes the mount. 


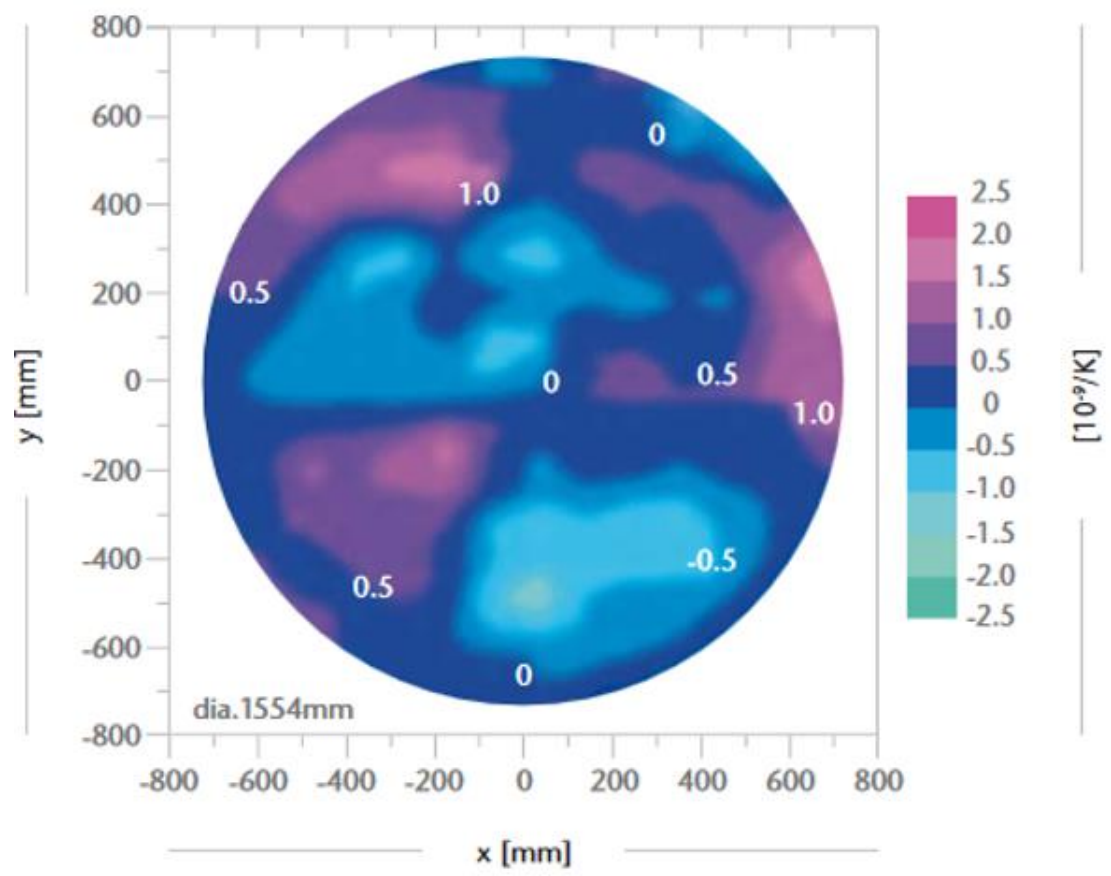

Figure 9: This is the CTE homogeneity of an example Zerodur boule. ${ }^{[1]}$

This distribution appears to have very low order spatial terms like astigmatism and coma, so code was written to produce CTE distributions of similar shapes. The P-V of the CTE distribution was limited to $5 \mathrm{ppb} / \mathrm{K}$ to be in line with data in Figure 10 that is from a paper published by Schott. ${ }^{[2]}$ These efforts resulted in a prediction that matched the measured data's RMS quite well as shown in Figure 11. Further analysis (described in section 4) is done to match the measured data's shape.

\begin{tabular}{|c|c|c|c|c|c|c|}
\hline & Dimension & $\begin{array}{c}\text { Number of } \\
\text { Samples }\end{array}$ & $\begin{array}{c}\text { CTE }\left(0^{\circ} ; \mathbf{5 0}^{\circ}\right) \text { absolute value } \\
{[\mathbf{p p b} / \mathbf{K}]}\end{array}$ & \multicolumn{2}{|c|}{$\begin{array}{c}\text { CTE }\left(0^{\circ} ; \mathbf{5 0}^{\circ}\right) \text { homogeneity } \\
{[\mathbf{p p b} / \mathbf{K}]}\end{array}$} \\
\hline Year & {$[\mathbf{m m}]$} & $\#$ & Specification & Achieved & Specification & Achieved \\
\hline 2003 & $4100 \times 171$ & 18 & $+/-50$ & 66 & 20 & $18^{1}$ \\
\hline 2005 & $3610 \times 370$ & 12 & $+/-100$ & 80 & 30 & $25^{1}$ \\
\hline 2009 & $3700 \times 163$ & 36 & $+/-150$ & 54 & 40 & 9 \\
\hline 2010 & $3400 \times 180$ & 12 & $+/-100$ & 42 & 30 & 5 \\
\hline 2012 & $4250 \times 350$ & 16 & $+/-30$ & 60 & 40 & 5 \\
\hline 2014 & $4250 \times 350$ & 16 & $+/-30$ & 0 & 40 & 3 \\
\hline 2016 & $4060 \times 103$ & 16 & $+/-50$ & 36 & 20 & 7 \\
\hline $2016^{2}$ & $4000 \times 100$ & 12 & $+/-150$ & 15 & 20 & 4 \\
\hline
\end{tabular}

Figure 10: This is an image of a table from a paper published by Schott. ${ }^{[2]}$ The first two measurements (circled in red) were taken using an old dilatometer setup. The newer, more accurate measurements indicate much better homogeneity which is clustered around $5 \mathrm{ppb} / \mathrm{K}$. 

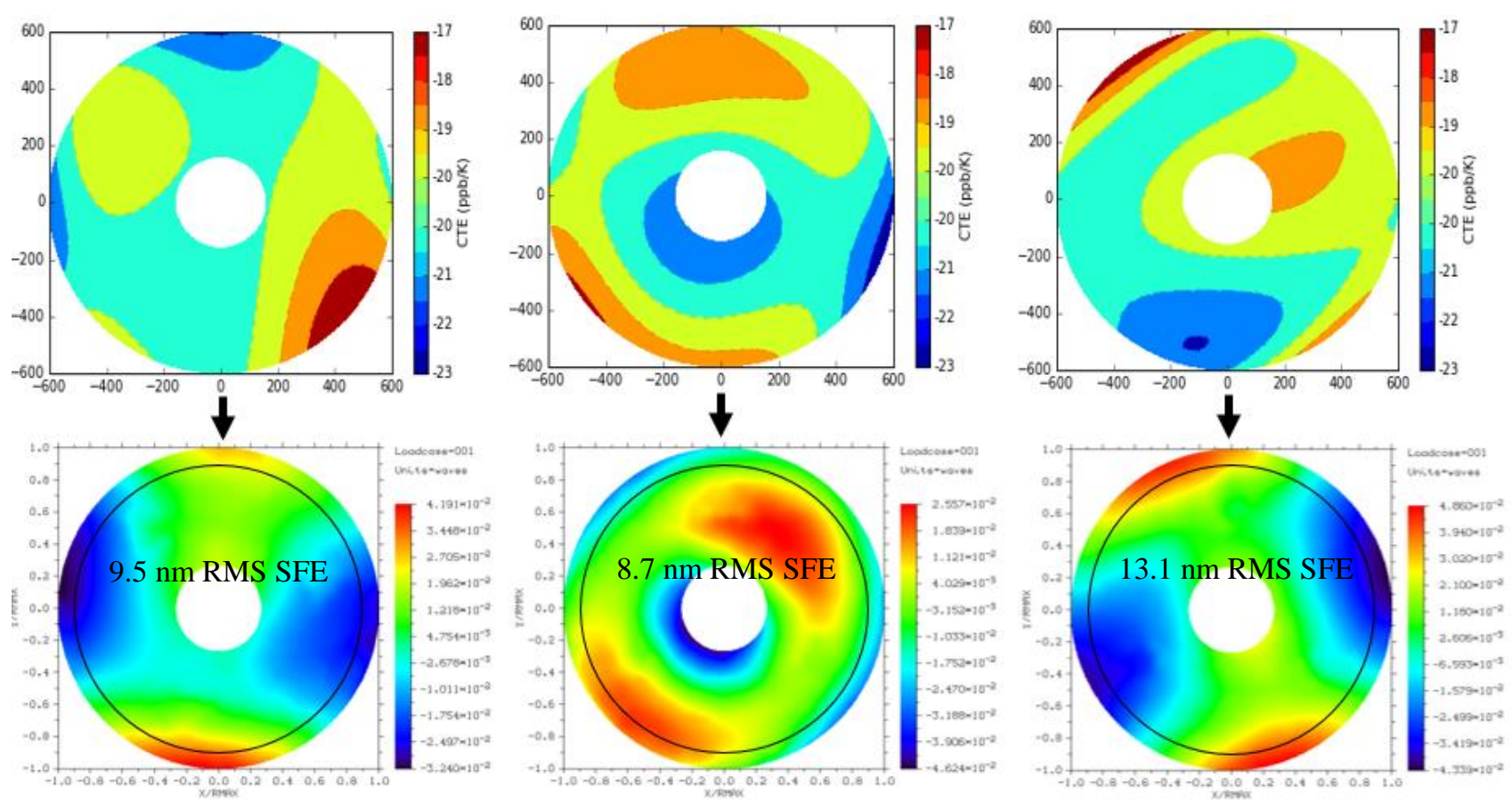

Figure 11: SFEs produced by random CTE distributions that have a 5ppb/K P-V are shown. The top row shows the CTE distributions and the bottom row shows the resulting SFE. The top left CTE distribution resulted in a SFE of 9.5nm RMS which is very similar to the measured SFE of $9.4 \mathrm{~nm}$ RMS. The high variation in RMS SFE with a constant P-V CTE implies that the P-V CTE is not adequate for estimating the performance of the optic, and thermal soak testing or knowledge and analysis of the CTE distribution is necessary. 


\section{CORRELATION OF MODEL TO TEST DATA}

The ELZM was produced by Schott as a demonstration of aggressive lightweighting, and these thermal tests were not anticipated. Therefore, the CTE of this Zerodur was not optimized for the test temperatures, and the CTE distribution for this was not measured. However, Schott has provided many literary resources that provide an expectation for the range of possible CTE distributions of the mirror. ${ }^{[1][2]}$ Because the CTE distribution is unknown, a correlation process has been used to correlate the thermoelastic model to the test data by tuning the CTE distribution. A byproduct of the correlation process includes information relating to the sensitivity of any mirror to a thermal gradient.

\subsection{Mathematical Process}

In brief: the RMS of the difference between the test data and analysis results is minimized by changing the CTE distribution of the mirror within reasonable bounds.

This is accomplished using the following steps:

1) Apply a CTE distribution to the mirror. The CTE distributions for this work were a summation of Zernike shapes with variation from case to case resulting from an intentional variation in the shape coefficients. Zernike shapes were used between standard Zernike number 4 and 91 (from $n=2, m=0$ to $n=12, m=12$ ). This process is described mathematically in equation (1) and graphically in Figure 12.

2) Run the mirror finite element model (FEM) through NASTRAN.

3) Run the displacements found with NASTRAN through SigFit to find the SFE after power and rigid body motions have been removed.

4) Subtract the analysis' surface figure error from the measured surface figure error to find the residual SFE.

5) RMS the residual SFE.

6) Calculate the objective function by combining the RMS residual SFE with a term that constrains the CTE distribution's $\mathrm{P}-\mathrm{V}$ to a realistic number.

7) Repeat steps 1-6 until the objective function reaches a minimum.

Producing a CTE distribution is central to these steps. Mathematically, this is described by equation (1).

$$
\left[\alpha_{x, y}\right]=\sum_{n=2}^{12} \sum_{m=0}^{n} C_{n, m}\left[U_{n, m}\right]
$$

Where $\alpha_{x, y}$ is the CTE distribution, $C_{n, m}$ is the coefficient for the Zernike shape which is represented with $U_{n, m}$. This summation is expressed graphical in Figure 12.
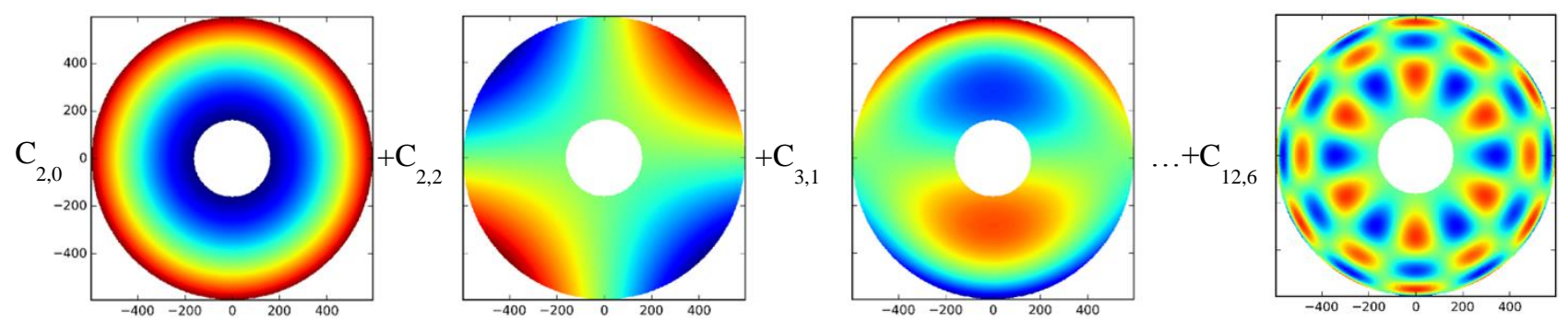

Figure 12: This figure shows a few of the Zernike shapes that are summed in the summation process which is mathematically described by equation (1). The coefficients $\left(\mathrm{C}_{\mathrm{n}, \mathrm{m}}\right)$ are multiplied with the Zernike shapes, and the resulting surfaces are summed to create a CTE map like what is shown in Figure 13. 

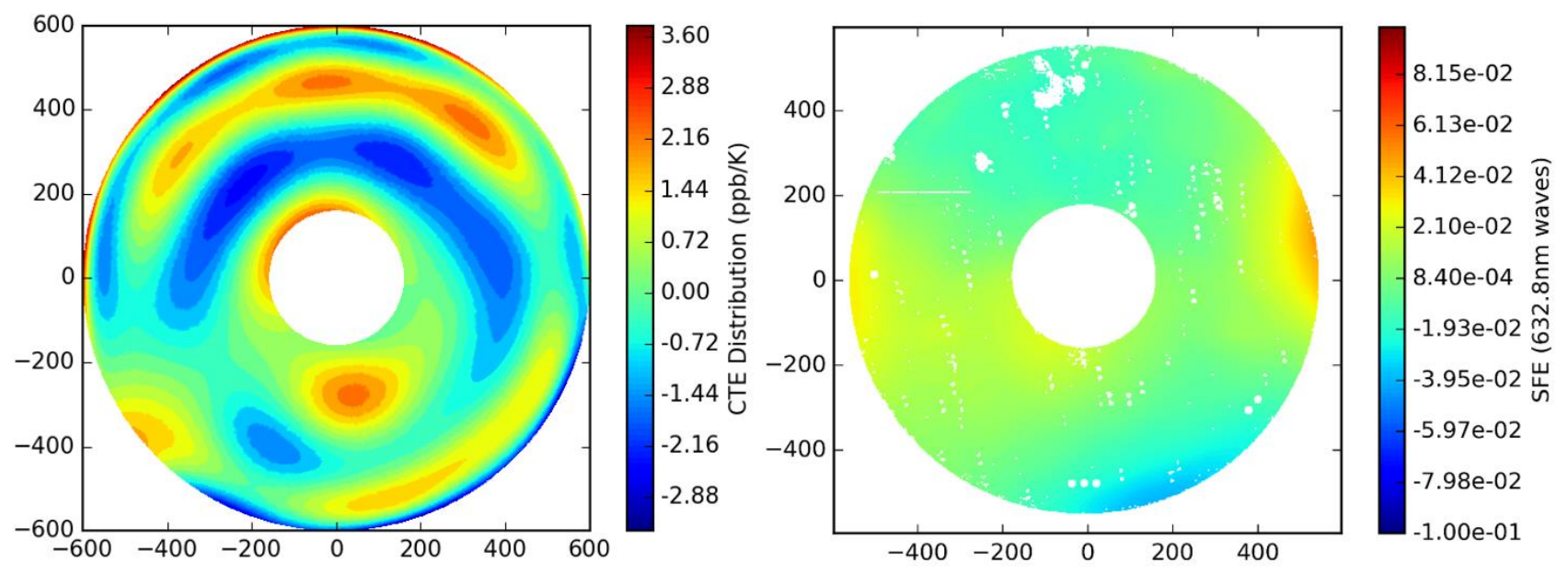

Figure 13: Left: A CTE distribution that was produced using the correlation process. Right: The SFE that this CTE distribution causes when the mirror's temperature decreases by $62 \operatorname{degC}$ and is filtered using the process described in section 2.2.

\subsection{Results from Correlation}

Several CTE distributions were produced that resulted in SFEs that closely matched the test data. Multiple CTE distributions cause the same SFE from a thermal soak. This is made true by the fact that some CTE shapes have a small impact on SFE (as discussed in section 4.3.), so the CTE distribution may change by a large amount without impacting SFE by more than the SFE measurement accuracy. Visual evidence of this provided in Figure 14.
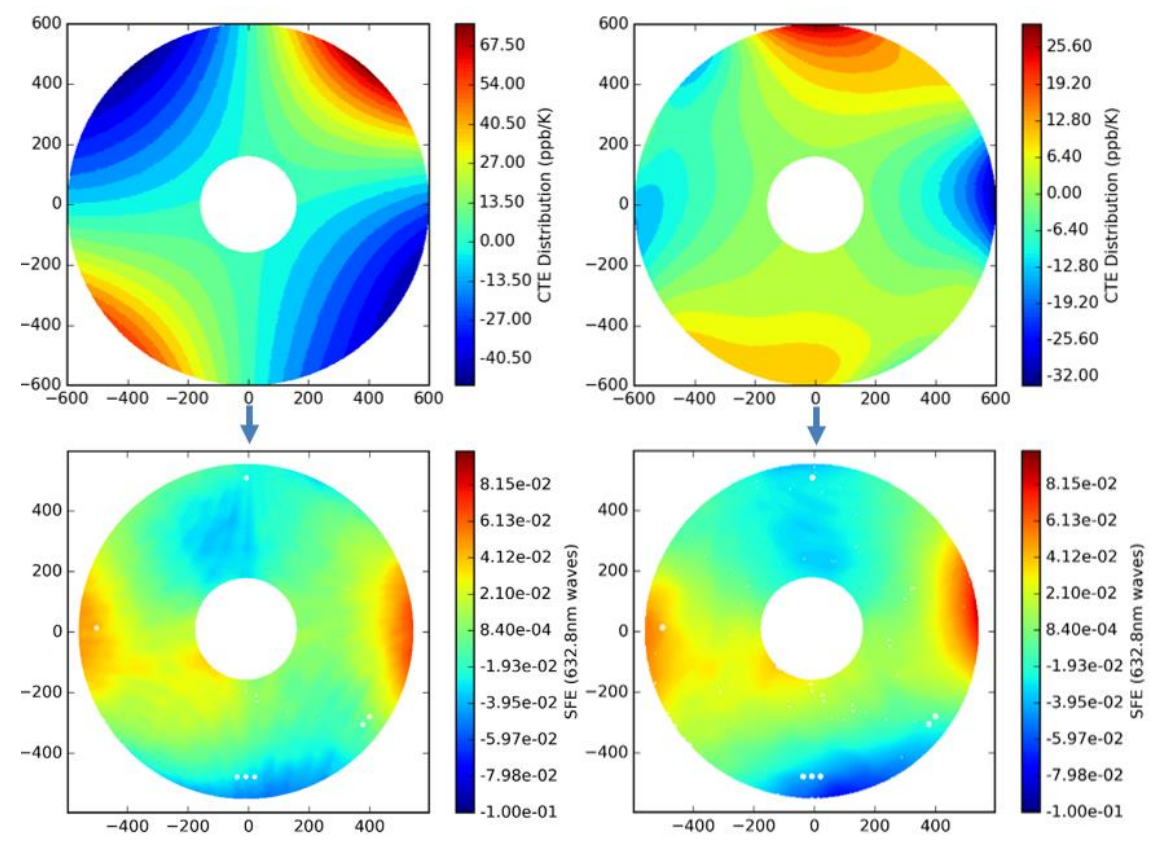

Figure 14: The top row shows the CTE distributions that result in the SFEs shown on the bottom row. The top left CTE distribution has a P-V of $110 \mathrm{ppb} / \mathrm{K}$. The top right CTE distribution has a P-V of $60 \mathrm{ppb} / \mathrm{K}$. Even though the CTE distributions are quite different in magnitude, the SFEs that result from these CTE distributions (and a $-62{ }^{\circ} \mathrm{C}$ change in temperature) are similar as is seen in the bottom row. This is because a first order astigmatism CTE distribution does not greatly affect SFE.

The two CTE distributions shown in Figure 14 are quite unrealistic, because their P-V is significantly greater than what Schott has measured in Zerodur boules. Therefore, a penalty was added to the correlation process's objective function for unrealistic CTE distributions, and the correlation algorithm resulted in the CTE distributions and SFEs shown in Figure 15 and Figure 16. The two CTE distributions match the measured SFE to within the repeatability of the SFE measurements. 
Both CTE distributions have reasonable P-Vs $(7 \mathrm{ppb} / \mathrm{K}$ and $3 \mathrm{ppb} / \mathrm{K})$, so they are both considered to be successful correlations.
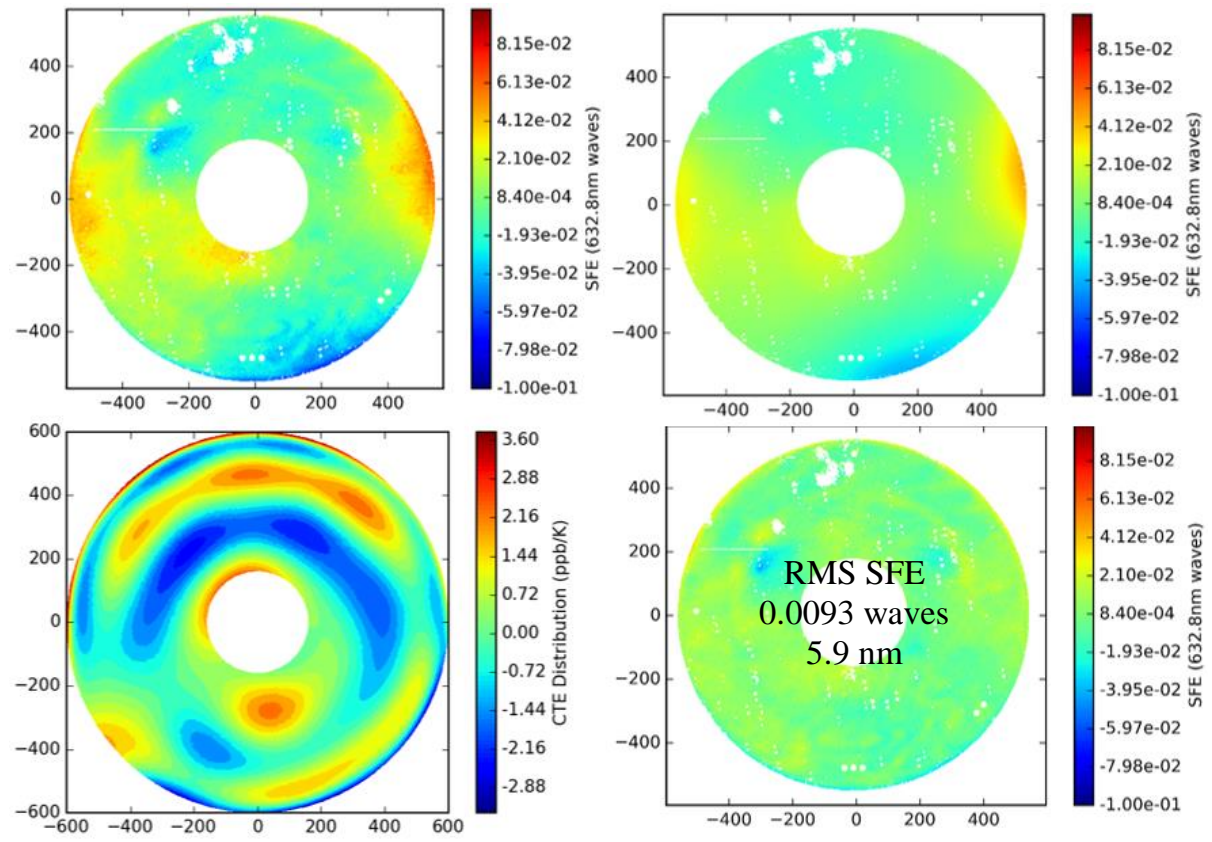

Figure 15: Correlation Results. The top left image shows the filtered, measured SFE for a 292K-230K soak. The top right image shows the filtered, analytical SFE for a $292 \mathrm{~K}-230 \mathrm{~K}$ soak. The bottom left image shows the CTE distribution that was used to obtain the analytical SFE. It has a P-V of roughly $7 \mathrm{ppb} / \mathrm{K}$ which is within the range of measured CTE distributions shown in Figure 9. The bottom right image shows the difference between the measured and analytical SFE. The RMS of the difference between the measured and analytical SFE is $5.9 \mathrm{~nm}$ which is better than the repeatability of the test, so the correlation process is considered to be successfully completed.
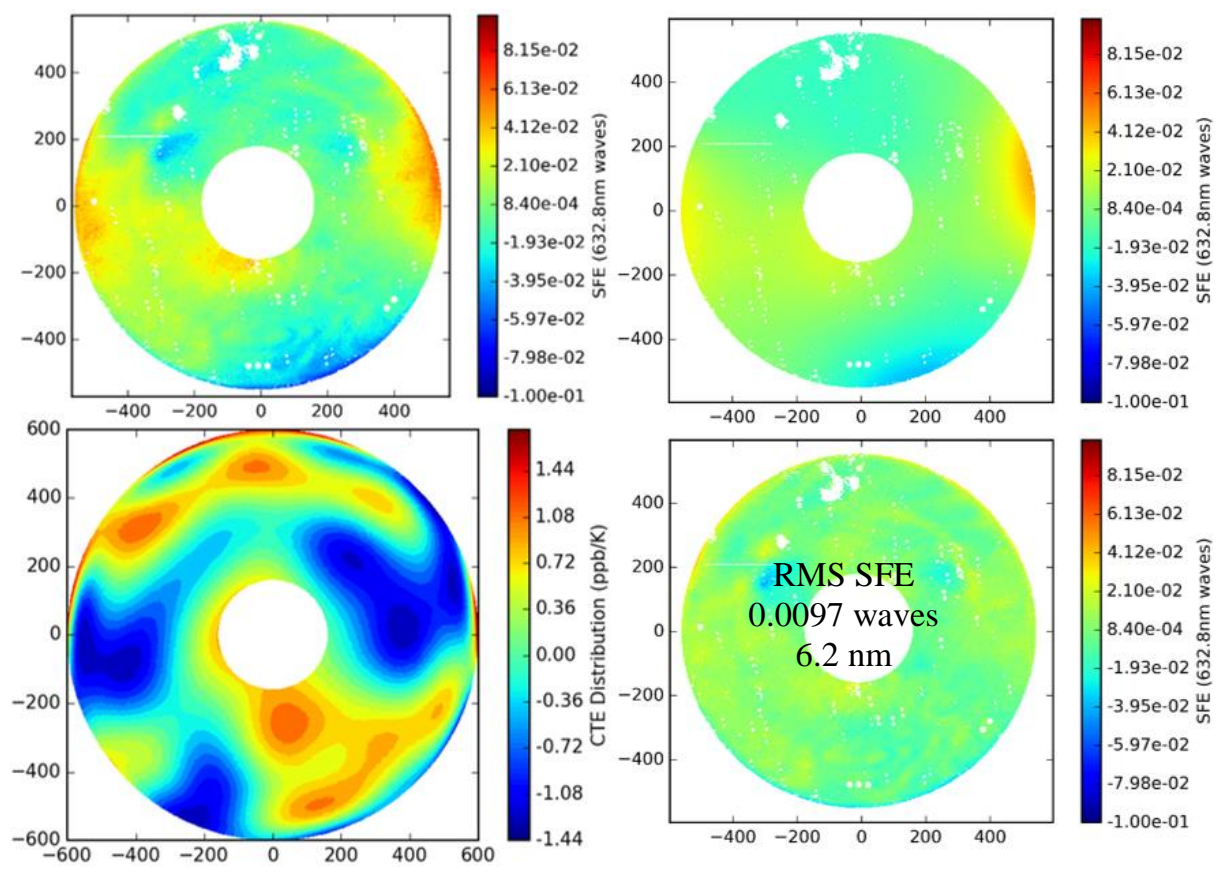

Figure 16: Correlation Results. The images are in the same order as is shown in Figure 15. The CTE distributions have some similarities, yet they are distinctly different and still result in similar SFE maps. This CTE distribution has a P-V of $3 \mathrm{ppb} / \mathrm{K}$ which is within the range of measured CTE distributions shown in Figure 9. 


\subsection{Sensitivity of SFE to several CTE distributions}

While following the mathematical process previously described in section 4.1, several interesting and useful sensitivities were determined. These sensitivities illustrate which temperature and CTE distributions will produce the greatest uncorrectable SFE. A mirror's temperature or CTE distribution may be very large, but if it is of a benign shape then its effect on SFE will be quite small. This is important for thermal analysts to understand in order to can see which thermal gradients cause significant SFE and which do not. Simply quoting the P-V of the temperature distribution (often called the temperature gradient) may result in an overly conservative or overly optimistic projection for optical performance.

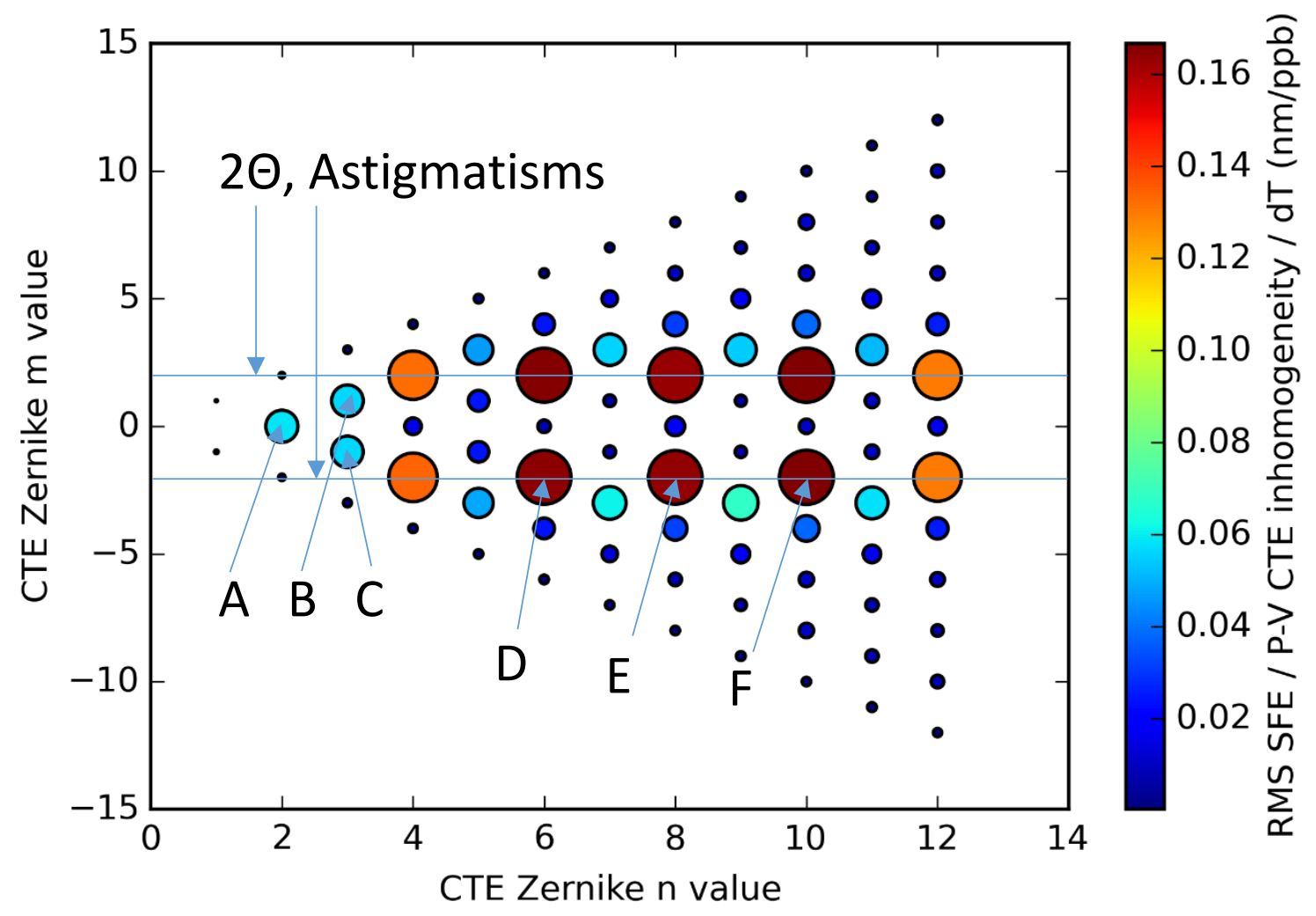

Figure 17: SFE Sensitivity to Zernike Shapes. The circles and colors represent the sensitivity of the SFE to a Zernike shape with that circle's $n$ and $m$ value. The Zernike shapes marked as A-F are highlighted in later figures.

Figure 17 provides a graphical representation of the RMS SFE sensitivity to CTE distributions that have the shape of the Zernike polynomials. These values were determined by individually placing $2 \mathrm{ppb} / \mathrm{K} \mathrm{P-V} \mathrm{CTE} \mathrm{of} \mathrm{each} \mathrm{Zernike} \mathrm{shape} \mathrm{onto}$ the mirror and increasing the mirror's temperature by $1 \mathrm{~K}$. Figure 17 shows that the most sensitive coefficients are $\sim 500 \mathrm{x}$ more sensitive than the least sensitive. The actual magnitude of these values will be dependent upon the mirror's geometry as defined by the equation in section 5.2.

The most commonly seen thermal gradients take on the shape of coma, defocus, and tip/tilt. Coma and defocus produce a moderate amount of SFE, but tip/tilt produces an insignificant amount. The errors caused by coma and defocus temperature gradients are shown in Figure 18. The most sensitive thermal gradient shapes are fortunately extremely uncommon. These shapes and the resulting SFE are shown in Figure 19. 

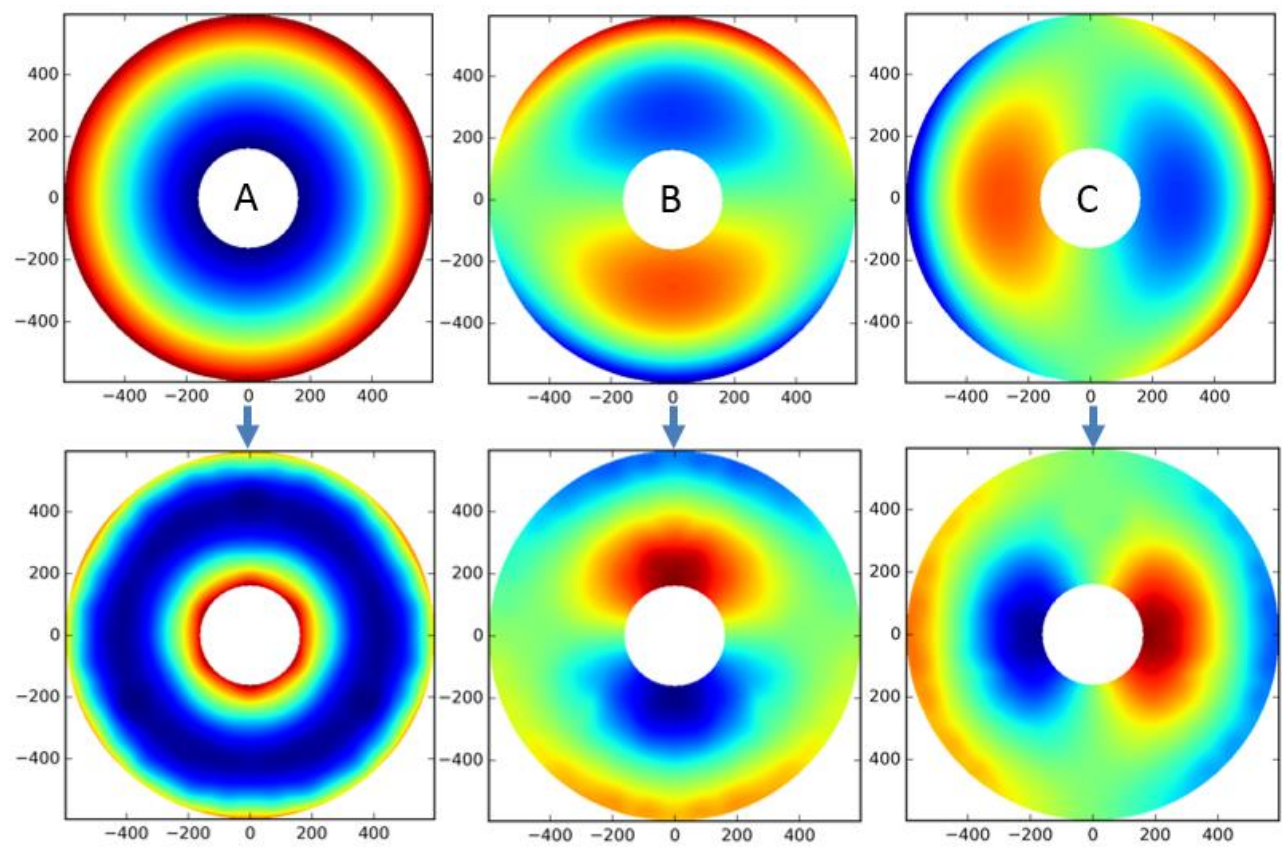

Figure 18: CTE or temperature distributions (top) and the resulting surface figure error (bottom). Temperature distributions that are in the shape of defocus (A) are commonly produced when the view factors around the mirror's sides and behind the mirror are at one temperature while the view factor in front of the mirror is at another temperature (like a mirror surrounded by a shroud and viewing deep space). Temperature distributions that are in the shape of coma (B \& C) are commonly produced when a telescope's side is illuminated by a significant heat source (like the sun). The asymmetry that is most apparent in B's SFE is due to the mirror's core structure which has three mounting zones at 0 degrees (above central hole), 120 degrees (to the bottom right of the central hole) and 240 degrees (to the bottom left of the central hole).
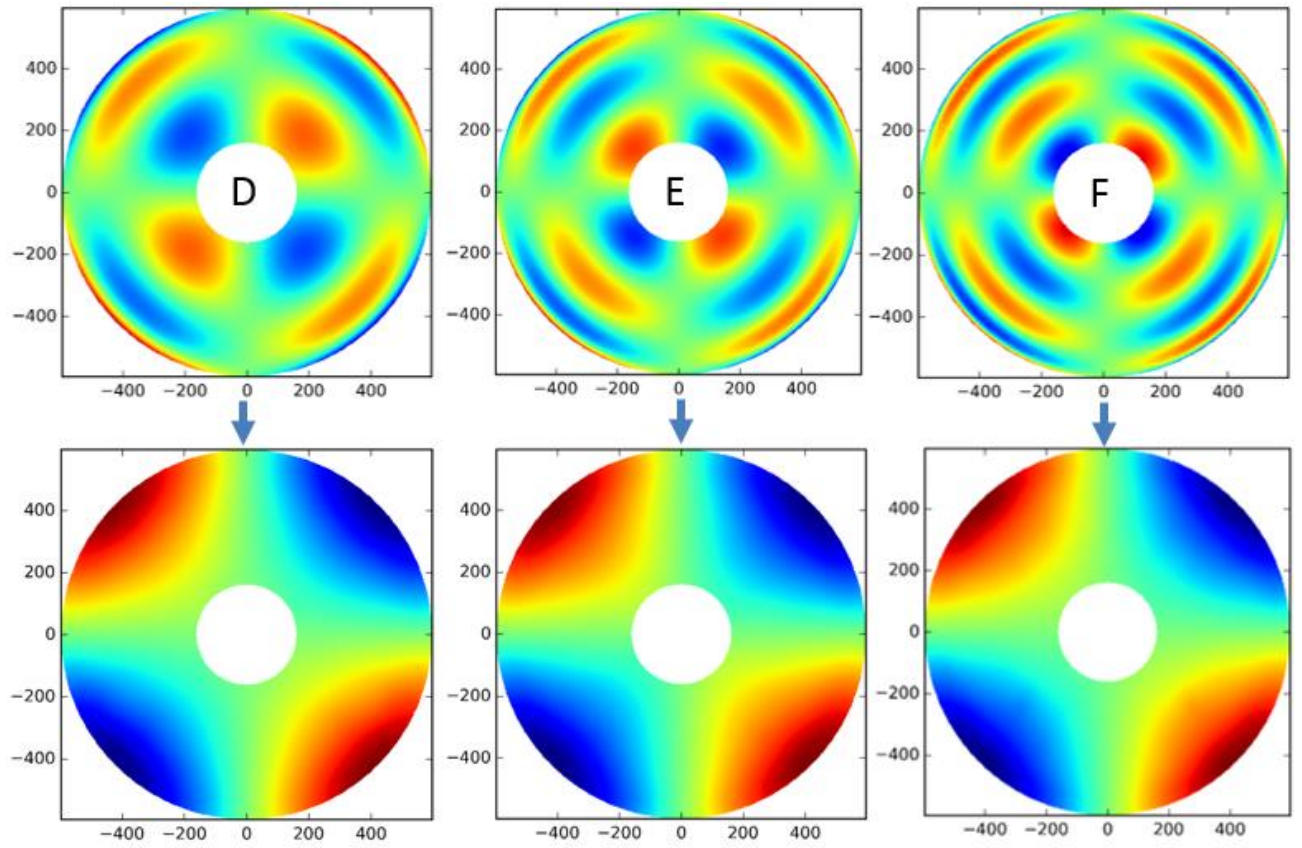

Figure 19: CTE and temperature distributions that result in a large uncorrectable SFE. The top row shows the CTE or temperature distribution and the bottom row shows the resulting SFE. Notice that the worst shapes are all 2- $\theta$ with several cycles per radius. It is extremely unlikely that a temperature distribution would exist with this shape, but some amount of this shape may be present in CTE distributions. All of these result in an astigmatism error which is the first bending mode of the mirror. Test of several mirror materials also indicates that CTE distributions typically cause astigmatism. ${ }^{[3]}$ 


\section{USE OF SENSITIVITY DATA FOR THERMAL GRADIENTS}

\subsection{Why the sensitivities work for thermal gradients}

A kinematically mounted mirror's SFE is affected by thermal in two ways: thermal gradients and CTE gradients. The test focused on the effect of CTE gradients; not thermal gradients, but a brief argument is presented below that shows the two are mathematically analogous. For the case of the cantilevered beam shown in Figure 20, the analogous nature of these two sources can be seen by taking the spatial derivative of the linear thermal expansion equation as shown below.

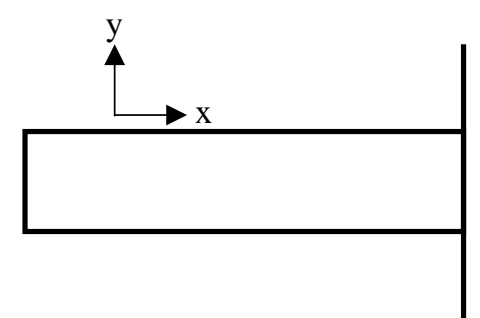

Figure 20: Simple 1D Rod

$$
\begin{gathered}
\varepsilon_{x}=\alpha \Delta T \\
\frac{d \varepsilon_{x}}{d y}=\frac{d}{d y}[\alpha \Delta T]
\end{gathered}
$$

For bending about $\mathrm{z}$ from thermal gradients in a completely homogeneous rod:

$$
\frac{d \varepsilon_{x}}{d y}=\frac{d \Delta T}{d y} \alpha
$$

For bending about $\mathrm{z}$ from CTE gradients in an isothermally soaked rod:

$$
\frac{d \varepsilon_{x}}{d y}=\frac{d \alpha}{d y} \Delta T
$$

Therefore, a rod that has a CTE gradient of $1 \mathrm{ppb} /{ }^{\circ} \mathrm{C} / \mathrm{m}$, which experiences a thermal soak of $1^{\circ} \mathrm{C}$, will deflect by the same amount as a rod that has a temperature gradient of $1{ }^{\circ} \mathrm{C} / \mathrm{m}$ and a CTE of $1 \mathrm{ppb} /{ }^{\circ} \mathrm{C}$. Likewise, the surface of a mirror that has a CTE distribution that is coma with $1 \mathrm{ppb} /{ }^{\circ} \mathrm{C} \mathrm{P}-\mathrm{V}$, which experiences a thermal soak of $1^{\circ} \mathrm{C}$, will change by the same amount as a mirror that has a thermal gradient that is coma with $1{ }^{\circ} \mathrm{C} \mathrm{P}-\mathrm{V}$ and a uniform $\mathrm{CTE}$ equal to $1 \mathrm{ppb} /{ }^{\circ} \mathrm{C}$.

To generalize these sensitivities even further, one must take into account the effect of the mirror's geometry on the SFE. A mirror's SFE change due to CTE homogeneity is directly proportional to the depth of the mirror. The generic sensitivities are shown in Table 1.

Table 1: Sensitivity of a mirror's SFE to various thermal disturbances of shapes defined by the Zernike polynomials. There is not perfect symmetry in these numbers, because the mirror's core structure had pad stiffening features that affected $-\mathrm{m}$ and $+\mathrm{m}$ terms differently.

\begin{tabular}{|c|c|c|c|c|c|c|c|c|c|c|c|}
\hline \multicolumn{10}{|c|}{ SFE Sensitivities (nm RMS SFE/(ppb/C)/( C)/m } \\
\hline $\mathbf{n} \backslash \mathbf{m}$ & $\mathbf{- 5}$ & $\mathbf{- 4}$ & $\mathbf{- 3}$ & $\mathbf{- 2}$ & $\mathbf{- 1}$ & $\mathbf{0}$ & $\mathbf{1}$ & $\mathbf{2}$ & $\mathbf{3}$ & $\mathbf{4}$ & $\mathbf{5}$ \\
\hline $\mathbf{1}$ & & & & & 0.007 & & 0.003 & & & & \\
\hline $\mathbf{2}$ & & & & 0.02 & & 0.47 & & 0.02 & & & \\
\hline $\mathbf{3}$ & & & 0.03 & & 0.45 & & 0.45 & & 0.03 & & \\
\hline $\mathbf{4}$ & & 0.03 & & 1.07 & & 0.13 & & 1.06 & & 0.03 & \\
\hline $\mathbf{5}$ & 0.03 & & 0.39 & & 0.19 & & 0.20 & & 0.38 & & 0.04 \\
\hline
\end{tabular}




\subsection{How to use the sensitivities for thermal gradients}

The equation to use the sensitivities for thermal gradients is shown below:

$$
S F E=\operatorname{ch} \alpha T_{p-v}
$$

Where SFE is the RMS SFE after removing power (nm), c is the SFE sensitivity coefficient found in Table $1, \mathrm{~h}$ is the mirror's depth $(\mathrm{m}), \alpha$ is the mirror's CTE $(\mathrm{ppb} / \mathrm{K})$, and $\mathrm{T}_{\mathrm{p}-\mathrm{v}}$ is the maximum minus the minimum mirror temperature $(\mathrm{K})$.

For example, consider the $2.5 \mathrm{~K}$ temperature gradient in the ELZM for the $230 \mathrm{~K}$ measurement. The ELZM has a varying depth, but it is near $0.125 \mathrm{~m}$. The temperature gradient is $2.5 \mathrm{~K}$. The gradient is mostly caused by the mount which attaches at three points resulting in a higher order trefoil shape $(\mathrm{n}=5, \mathrm{~m}=3)$. The mirror is made out of Zerodur which has a CTE of around $20 \mathrm{ppb} / \mathrm{K}$. The RMS SFE (after removing power) is calculated with equation 5:

$$
\begin{gathered}
S F E=\operatorname{ch} \alpha T_{p-v} \\
S F E=0.39 * 0.125 * 20 * 10^{-9} * 2.5\left[m \frac{1}{{ }^{\circ} \mathrm{C}}{ }^{\circ} \mathrm{C}\right]=2.43 \mathrm{~nm} \text { RMS SFE }
\end{gathered}
$$

Therefore, the error due to the thermal gradient in the thermal soak test is small relative to the error due to the thermal soak. If the same problem were presented except the temperature distribution in the mirror were only a lateral gradient, then the resulting RMS SFE would be greatly reduced by somewhere around 60-150x (based upon the ratio of sensitivities), because the sensitivity of SFE to lateral gradients is very low as seen in Table 1. However, when there is a lateral temperature gradient, there is often a coma temperature gradient present as well.

This method is best used when the thermal gradient's shape is very similar to a Zernike shape. Worst case assumptions can also be used to find a SFE that the mirror is certainly not exceeding. For example, if there is a strong lateral gradient that is potentially masking a coma gradient then the analyst may assume a coma gradient that is larger than what the observed lateral gradient could mask. If high accuracy analysis is required then it is best to use conventional numerical structural, thermal, optics (STOP) analysis approaches.

\section{CONCLUSION}

In conclusion, thermal soak testing has been done on an extremely lightweight Zerodur mirror. Models were successfully correlated to test data. The model correlations agree with Schott's data on the measurement of Zerodur's homogeneity. ${ }^{[2]}$ CTE P-V is often used for material characterization, but it does not constrain the performance of an optic, because some CTE distributions are benign (tip/tilt) compared to others (2-theta with several cycles per radius). MSFC's testing campaign will quantify materials by determining the RMS SFE produced per degree change in temperature per mirror depth. The correlation process has also produced the first hand calculations to estimate surface figure error due to coefficient of thermal expansion distribution or temperature distributions.

\section{REFERENCES}

[1] Schott, "ZERODUR ( ${ }^{\circledR}$ Zero Expansion Glass Ceramic," Schott website, July 2011, http://www.schott.com/d/advanced optics/f7ae3c11-0226-4808-90c759d6c8816daf/1.0/schott zerodur katalog july 2011_en.pdf (July 2011).

[2] Jedamzik, Ralf, et al., "Effects of thermal inhomogeneity on 4m class mirror substrates", Proc. SPIE 9912, Advances in Optical and Mechanical Technologies for Telescopes and Instrumentation II, 99120Z (July 22, 2016); doi:10.1117/12.2234287; http://dx.doi.org/10.1117/12.2234287

[3] Smith, W. Scott, et al., "Mirror Requirements for Far-IR \& Sub-MM," Proc. From Spitzer to Herschel and Beyond: The Future of Far-IR Space Astrophysics International Conference, Session K (2004). 